\title{
Research on Game Mode and Tactical and Technical Analysis Based on SPSS Method
}

\author{
Lin Zuo ${ }^{1, a}$, Chunsheng $\mathrm{Li}^{1}$, Xiong $\mathrm{Liu}^{2}$ \\ ${ }^{1}$ Wuyi University, Jiangmen, China \\ ${ }^{2}$ Jiangmen Waihai Middle School, Jiangmen, China \\ alin_zuo88@163.com
}

Keywords: defense force; time and space force; control force; attacking force; game mode; tactics; multivariate analysis

\begin{abstract}
With the development of football in constant change and improvement, China has payed more attention to football match. However, game modes and technical and tactical occupy a vital position in football game. It directly affects the football talents training mode and professional league's level of the whole nation. So ,successful organization ,skillful game mode application and tactics is the important factor attributing to a triumph in a match. This paper focus on determining the research object and research method, using multivariate analytical method to analyze the team game mode and further use mathematical modeling thought deepening technical analysis of the 11th national games men's football in Guangdong province. The analysis results show that four factors play a leading role in the match ,they are defensive force, time and space usage force, controlling force and offensive force. It can provide certain theoretical basis and the practical path for analyzing and diagnosing football game mode and technical analysis.
\end{abstract}

\section{Introduction}

Stepping into the 21st century, China's attention towards soccer strategy and tactics has increased day by day .A lot of scholars and football training education teaching workers do relevant theory analysis research in this field, such as Zhang Qingchun[1] .He did analysis of general characteristics and technique and tactics characteristics about men's football finals in the 2004 Summer Olympics in Athens. It mainly depends on features of tactical analysis on the whole process in man football finals in the 2004 Olympics in Athens, based on all the events of overall viewing, analysing player 's individual characteristics of World Team national team, highlighting the principal part's characteristics and incorporated into the overall strategic and tactical analysis. In addition, Wang Xinghua's Q-type Cluster has comprehensively evaluated each football teams ' technique and tactics ability of the 17th World Cup ,which is mainly quoted Q-type Cluster Analysis principal.

At first, Record every football team's comprehensive strength in the 17th World Cup ,and then according to the live, a useful and effective team technical and tactics ability has worked out aimed at doing empirical analysis towards the competition strategy and tactics. As above, there are more analysis researches in techniques and tactics aspects in a football match. On the other hand, researches which are based on techniques and tactics of big-game-mode are relatively less[2].This paper is based on the football match model, making use of many kinds of analysis method to study them so as to achieve more scientific and reasonable recommendations on further analysis of football technical and tactical ,to broaden thinking for this project's research, to explore more human method and provide feasible lessons for our nation's football career[3].

\section{The research objects and methods}

A. Research object

This paper researches and analyzes 6 matches of 1st men's football team behaving on the 11th national games in Guangdong province.It also videos football game match action which is broadcasting on Shangdong Satellite.

B. Research method 
(1)Literature survey

Consult and collect domestic or foreign article, book and journals.

(2) Field survey method

Do field survey about the whole 6 matches process of 1st men's football team ,and video them.

(3)Interview survey method

As far as big game mode, tactics and other problems, the Guangdong team manager Mr. CaoYang, the captain Mr. Guang Zhirui, the Guangdong province sports bureau deputy director Mr. Ye Xiquan and Mr. Zhao Shaoming are interviewed, the first-hand information are gotten.

(4)comparative analysis approach

During competing with other teams, some related datum are researched and analyzed.

(5)Video statistics

According to indexes such as the offensive way of football, offensive and defensive system formation characteristics, do video observation statistics, refer to various statistical index to do comprehensive evaluation analysis[4].

(6)Mathematical statistics

Make use of the SPSS 13.0 software and statistical method to collect data for statistical analysis processing

\section{The result and analysis}

A. The main formation in match

The "four-four-two" formation is made up of 4 midfield ,4 full backs and 2 strikers. Because 4 full backs are used, this formation can change variously in midfield."Four -four- two" formation is adopted when competing gainst Liaoning team this time.

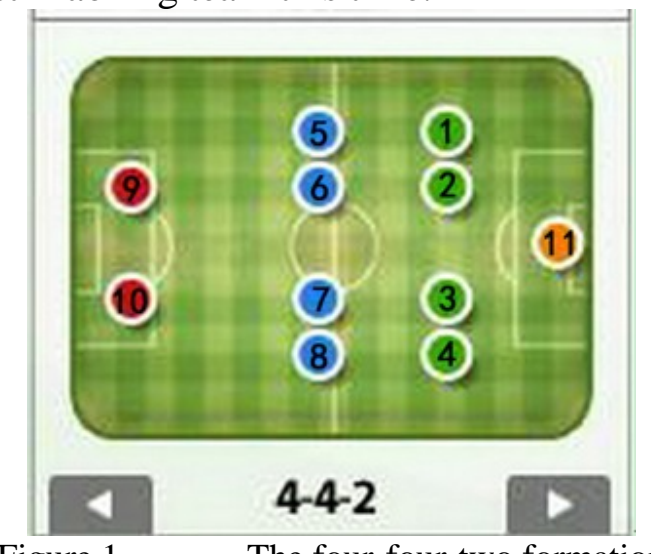

Figure 1.

The four-four-two formation

(Note: 1 Chen Jianlong 2.Xu Weilong 3.Liu yuchen 4.Chen Haibing 5.Pan Jia 6.Yu Jianfeng 7.Yin Hongbo 8.Yin Hongbo 9.Ye Weichao 10.Shi Liang 11.Guo Zichao)

Strikers are on both sides or one side and middle side, or strikers are at the back and in the front,or diamond-shaped arrangement .Under the blanket defence, at the same time, they try best to fight for the right of drop-ball[5].Two strikers insert forward and back, run off the ball actively, attract defence, manufacturing neutral position and opportunity, making full use of the personal ability and under the corporation of teammates so as to break the defence of the opposing.In using this formation ,Guangzhou team adjusts specific tactical strategy according to the opponent's tactical strategy. When competing with Liaoning team and Shanghai team, the Guangzhou team chiefly strengthens the backline, consolidating step by step and implementing side way offensive way. However, their strategy is based on a solid defense to quickly counterattack when competing against Beijing team, Shandong team and Sichuan team.

B. The results and analysis of 13 technical index characteristic

The football team tactics style characteristics embodied in the specific tactics statistical index characteristics is closely linked to the result of the competition. Figure 2 is the 13 technical index's statistical differences under three situations: victory, defeat and draw of Chinese team on six matches. It comes to a conclusion: when goals scored $(\mathrm{p}<0.05)$, other indexes have no significant difference[6].Ultimately ,mainly because the number of negative field and flat field is almost 
same ,larger error would appear if One-way ANOVE is chose .In some information about the analysis of the material in football technical indicators, It also indicates that it perhaps unreasonable to decide win or lose just depending on the average size of index .This paper analyses three situations: victory, defeat and draw of six matches through basic datum ,and respectively draws out the advantage and disadvantages[7].

TABLE I. The statistics of relationship between 13 technical index and match results in the 11th national games men's football in Guangdong province

\begin{tabular}{|c|c|c|c|c|}
\hline Index & $\begin{array}{l}\text { Defeat } \\
\bar{X} \pm \mathrm{SD}\end{array}$ & $\begin{array}{c}\text { Draw } \\
\bar{X} \pm \mathrm{SD}\end{array}$ & Victory $\bar{X} \pm \mathrm{sD}$ & $\begin{array}{c}\text { Inspect } \\
\bar{F} \bar{P}\end{array}$ \\
\hline$X 1$ & $40.05 \pm_{19.587}$ & $52.41 \pm 6.081$ & $50.977 \pm 10.256$ & $1.343 \quad 0.293$ \\
\hline$x 2$ & $418.5 \pm 108.2$ & $448.0 \pm 115.7$ & $488.3 \pm_{144.5}$ & $0.312 \quad 0.737$ \\
\hline$x 3$ & $0.5 \pm 0.71$ & $0.67 \pm_{0.82}$ & $2.33 \pm 1.0$ & $\begin{array}{ll}7.336 & 0.007\end{array}$ \\
\hline$X 4$ & $418.5 \pm_{108.2}$ & $448.0 \pm_{115.7}$ & $488.3 \pm_{144.5}$ & $\begin{array}{ll}0.312 & 0.737\end{array}$ \\
\hline$X 5$ & $12.5 \pm_{12.02}$ & $18.833 \pm 6.882$ & $21.556 \pm 7.633$ & $\begin{array}{ll}1.145 & 0.346\end{array}$ \\
\hline$x 6$ & $4.5 \pm 4.949$ & $5.667 \pm 3.502$ & $5.778 \pm 2.682$ & $0.133 \quad 0.876$ \\
\hline$X 7$ & $2.0 \pm 0$ & $4.0 \pm 2.683$ & $3.111 \pm 3.218$ & $0.391 \quad 0.648$ \\
\hline$X 8$ & $19.0 \pm 4.243$ & $12.0 \pm 3.899$ & $12.778 \pm_{3.768}$ & $\begin{array}{ll}2.641 & 0.108\end{array}$ \\
\hline$X 9$ & $1.0 \pm_{0}$ & $1.334 \pm 0.817$ & $2.0 \pm_{1.119}$ & $\begin{array}{ll}1.338 & 0.294\end{array}$ \\
\hline$X 10$ & $10.0 \pm 2.828$ & $8.333 \pm 3.389$ & $8.667 \pm 2.121$ & $\begin{array}{ll}0.290 & 0.753\end{array}$ \\
\hline$X 11$ & $19.5 \pm 4.95$ & $18.333 \pm 7.090$ & $24.779 \pm 8.408$ & $\begin{array}{ll}1.351 & 0.291\end{array}$ \\
\hline$X 12$ & $13.0 \pm 2.828$ & $14.667 \pm 5.574$ & $19.222 \pm 8.258$ & $\begin{array}{ll}1.057 & 0.368\end{array}$ \\
\hline
\end{tabular}

(Note: X1-possession percentage;; X2-shots; X3-goals scored; X4-passes; X5-cross number ; X6-corner kick; X7-offside; X8-fouls committed; X9-res and yellow card ; X10-front free kick; X11-head success number; X12-Steals success number; X13- Scored frontcourt $30 \mathrm{~m}$ )

Table 2 lists the common factor's score coefficient ,so the function expression of common factor's score coefficient can be researched.13technical index X1、X2、X3、X4、X5、X6、X7、 X8、X9、X10、X11、X12、X13 are normalized values of Std. That is:

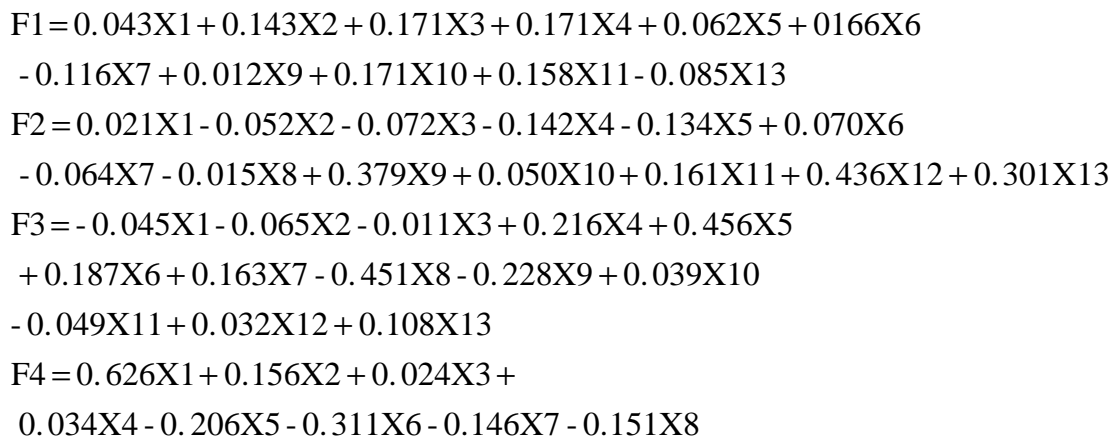

This computational formula indicates factor analysis of the whole process. The former load factor 's variables makes sure that 4 common factors are not ensured. As a result, it couldn't be common factor, so component matrix varimax is made, namely orthogonal rotation method. These informations could be got after load factors has rotated.

Constancy factor (F1) is mainly composited by probability of cross index ,possession percentage and scored frontcourt $30 \mathrm{~m}$. On the process of the match,the height of possession percentage directly affects the match result .The rest indicators are able to specifically perform the ability of game controlling in certain extent, they are main factors which affect match result on the whole match. Here, the first constancy factor can be brought into game control factor.

Constancy factor(F2) mainly consists of yellow and red cards, steals, foul index, directly express the defensive behavior. So, the second constancy factor can be brought into game defensive factor. Constancy factor (F3) mainly consists of offside, shots, corner kick and front free kick. These combination index is closely related to all the offensive behavior. So, the third constancy factor can be brought into game offensive factor.

Constancy factor (F4) consists of the combination of goal, pass and scored frontcourt $30 \mathrm{~m}$. These combination index is the precise performance of reasonably using space. Here, the fourth constancy factor can be brought into space utilization factor. 
C. The result of the match and running level analysis

1) Use running distance to analyze running level

The analysis of running level indicates that footballer must be capable of ceaselessly running 9$12 \mathrm{~km}$, which requires high-intensive physical ability. Figure 2 lists the running level category of footballers during the competence against Guangdong team and Liaoning team as well as the average of frequency of occurrence. The total distance that team members except goalie has ran includes :26\% of walking,38\% of jogging,13\% of full-speed sprint and $8 \%$ of move back running. But sideward running and oblique movement hasn't been listed in the category.

TABLE II. All running distance types and frequency of occurrence of each match(The number of people is 11)

\begin{tabular}{|c|c|c|c|c|}
\hline \multirow[t]{2}{*}{ Running types } & \multicolumn{2}{|c|}{ Running distance (meter) } & \multicolumn{2}{|c|}{ Frequency } \\
\hline & Average number & Standard deviation & Average number & $\begin{array}{l}\text { Standard } \\
\text { deviation }\end{array}$ \\
\hline Jogging & 1593 & 373 & 119 & 24 \\
\hline Hign speed run & 905 & 205 & 57 & 8 \\
\hline Sprints & 487 & 123 & 31 & 7 \\
\hline Walking & 1075 & 235 & 154 & 24 \\
\hline Move back & 279 & 123 & 60 & 18 \\
\hline Total & 4340 & 505 & 421 & - \\
\hline Playing distance & 79 & 42 & - & - \\
\hline
\end{tabular}

2) Analyze running level according to time

The time which players stand is $17.1 \pm 1.5 \%$ (Average number \pm error) by observing six matches of Guangzhou team, walking is $40.4 \pm 1.6 \%$.low intensity movement is $35 / 1 \%$,jogging is $16.7 \pm 2.3 \%(8 \mathrm{~km} / \mathrm{h})$,low speed running is $17.1 \pm 2.5 \%$,move back running is $1.3 \pm 0.3 \%$ among them high intensive running is $8.1 \%$,Medium speed run is $5.3 \pm 0.4 \%$,high speed run is $2.1 \pm 0.2 \%$ sprints is $0.7 \pm 0.1 \%(30 \mathrm{~km} / \mathrm{h})$ among them. As shown in Figure 2:
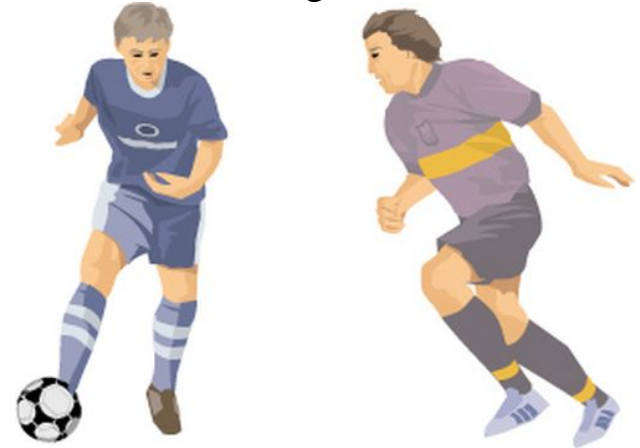

Figure 2. $\quad$ Example of intensity running and low-intensity running

3) Running level reflected in the results of the competition

Through research and analysis of the six games of the Guangdong team men's soccer , the Guangdong team can qualify for the National Games men's soccer final , and defeated Liaoning team at 8:7, winned the Eleventh National Games runner-up , which mainly thanks to its defense force and the use of time and space force, but control and offensive force less impact on competition. Figure 3 shows that jogging , backward and moving around has played an important role in defense force and played a crucial role in the game with the the Liaoning team and the Beijing team. Highspeed run , sprint and jogging mainly header, passing, scoring, in addition, the combination of events and space to play a powerful factor in promoting the time and space use force[8] .This factor is mainly reflected in the Battle with the Sichuan team and the Beijing team. And in competition with the Shanghai team , despite more use of control and offensive force, but the results are poor , eventually edged out $0-3$ to lose the title .

TABLE III. Figure 3 Relationship between result and running level

\begin{tabular}{|c|l|l|l|l|l|}
\hline $\begin{array}{c}\text { percentage of the common factor } \\
\text { in the race conditions }\end{array}$ & \multicolumn{5}{|c|}{ Running level } \\
\hline & Jogging & High speed run & Sprints & Walking & Move back \\
\hline Defence force & +++ & 0 & - & ++ & ++ \\
\hline Time and space use force & + & +++ & ++ & - & 0 \\
\hline Control force & ++ & + & - & 0 & + \\
\hline Offence force & 0 & + & ++ & - & - \\
\hline
\end{tabular}

(Note : +++ represents having very positive impact on match ability;++ represents having great positive impact on match ability;+ represents having little positive impact on match ability; 0 represents having no impact on match ability; - represents having little negative impact on match ability) 


\section{Conclusion}

Game mode and the technical and tactical characteristics of the Eleventh National Games in Guangdong football team is mainly attributed to the four aspects : offensive force , time and space use of force , control and defense force.The four factors determine the men's soccer team integrated technical and tactical, affecting the team's overall race results .Defense and space use force is very important to game, however ,control and defense force is relatively less important .According to the different characteristics of the players ,using the "4-4-2 " formation game in this tournament, the Guangdong team uses the best technical and tactical decision-making , which especially have made important contributions to the game 's achievements .

\section{References}

[1] Song Peng Luo Jiong.New Session of The Chinese National Men's Soccer Team Technical and Tactical Characteristics Research .China Sport Science and Technology,2010(6):77-79.

[2] Jin Chuanjiang,Cai Zhongjian,Lv Shengjun,Wang Xinhua.Application of Q -type Clustering of The 17th World Cup Comprehensive Evaluation of The Technical and Tactical Ability of Each Team. Journal of Beijing Sport University 2009 ,7(4):103-104

[3] Zhang xiaoquan, Wang xinsheng.The 9th National Women 's Football and Tactics CharacteristicsAnalysis. Journal of Anhui Sports Science. 2002, 06(2):75-78

[4] Li Qiang,Han Yu Heritage and Development of National Football Team Tactics Style . Sports Culture Guide.2011， 08 (8)

[5] Zhong Jianming,Jia Hong,Yang Xiaohong.Effect on high level football tournament factors of a multivariate statistical application research. Journal of Guangzhou Sport University, 2012,05(3):2325.

[6] Pan Changlei. Apriori Algorithm in Football Technical and Tactical Analysis. Computer Knowledge and Technology,2010，06(31):59-62

[7] Zhang qingchun,Liu Xianzhong ,Li Jianli,Liu Wenjuan. The 2004 Athens Olympic Games Men's Football Final General Characteristics and Technical Characteristics Analysis. Journal of Hebei Institute of Physical Education,2011,12 (4) :37-39

[8] Jin chuanjiang. Eighteenth World Cup footballMatch Technical Statistics Data and The Result of The Match Correlational Study. Chengdu Sport University,2010（1）:105-106. 